\title{
Scanning Atom Probe Analysis Alternately Triggered by Voltage and Laser Pulse*
}

\author{
Masahiro Taniguchi ${ }^{\dagger}$ \\ Department of Applied Chemistry, College of Bioscience and Chemistry, \\ Kanazawa Institute of Technology, 7-1 Ohgigaoka, Nonoichi, Ishikawa 921-8501, Japan \\ Osamu Nishikawa \\ Division of Industry-University Collaboration, Kanazawa Institute of Technology, \\ 7-1 Ohgigaoka, Nonoichi, Ishikawa 921-8501, Japan \\ (Received 7 December 2015; Accepted 22 February 2016; Published 12 March 2016)
}

\begin{abstract}
Voltage pulse which has been used from the establishment of atom probe (AP) in the 1960's is going to be replaced by short pulsed laser with high repetition rate in the nanometer scale characterization of materials by $3 \mathrm{D}-$ AP. Scanning Atom Probe (SAP) has a miniature electrode for acceptance of non-needle shaped specimens which allows us to analyze non-solid materials which are common in chemistry. The selectivity and the adaptability of voltage pulse and laser pulse are not clear in the non-bulk materials which are common in chemistry but not familiar in AP field. Two types of trigger, voltage pulse and laser pulse, were applied alternately to the identical sample to perform direct comparison. Tungsten tip, conventional material in AP, and amino acid (L-Tryptophan) supported on carbon nanotube were chosen as candidates for this analysis. The dual trigger system successfully worked on metallic sample and the selectivity of the ion species and adaptability were demonstrated on molecular system.
\end{abstract}

[DOI: $10.1380 /$ ejssnt.2016.69]

Keywords: Field ion microscopy; Field evaporation; Field ionization; Scanning atom probe; Mass spectroscopy; Tungsten; Biological molecules

\section{INTRODUCTION}

The authors have been working for the application of atom probe (AP) to the analysis of non-metallic materials such as graphite, carbon nanotubes (CNT), carbon fibers, and small molecules [1-3]. The introduction of local extractor electrode reduced the difficulty of fabrication of long sharp needle of these materials [4]. The unique capability of SAP has enabled us to analyze molecular system such as amino acid supported on single walled CNT (SWCNT) [6, 7].

The development of tomographic-AP (APT or 3D-AP) after the 2000's equipped with 2D position sensitive detector and short pulsed laser with high trigger rate realized atom-by-atom analysis of solid materials with 3dimensional real space information of nanometer scale [8].

The field evaporation triggered by laser pulse of visible $\sim$ infrared region of femtosecond time scale could be interpreted as the thermal pulsing coupled with rapid cooling. The application of voltage pulse is simply understood as the modulation of electric field. Though the two triggering method could be explained by the same model dealing with the change of potential diagram under electric field and the vibration of the surface atom, the differences of analytical results such as the mass spectrum (charge state), shadowing effect, etc. are still under discussion [9-11].

Though AP has the highest detection efficiency as analytical method, field evaporation is destructive process in atomic scale. Therefore, it is difficult to compare two different method under identical condition on the same

\footnotetext{
* This paper was presented at the 10th International Symposium on Atomic Level Characterizations for New Materials and Devices '15, Shimane Prefectural Convention Center (Kunibiki Messe), Matsue, Japan, October 25-30, 2015.

$\dagger$ Corresponding author: taniguchi@neptune.kanazawa-it.ac.jp
}

sample in atomic scale. To make the difference of the two triggering method clear, the authors examined the application of the two trigger alternately on the identical sample in this study. The two types of trigger, voltage pulse and laser pulse, were applied alternately to bulk material (tungsten tip) and molecular sample (amino acid) supported on carbon material. The mass spectra obtained by two trigger from identical sample were compared for the two cases.

\section{EXPERIMENTAL}

\section{A. Instrument}

The fundamental feature of scanning atom probe is described elsewhere $[4,5]$. The schematics of the instrument used in this work is shown in Figure 1. The specimen is positioned in front of the small probe hole of the miniature electrode by XYZ-stage with inertial drive piezo actuators. The time of flight and the hit position of field evaporated ions are detected by delay-line type 2D-position sensitive detector by Ionwerks of USA ("Meander"). The start (trigger) time and stop (hit) time are recorded by digital timer of 25 ps time resolution (TDC2PCI, Roentdek, Germany). Both control/data acquisition and data analysis program are written in our laboratory.

In the common setup, the pulse field evaporation of the positively DC biased specimen is driven by superimposing voltage pulse or by irradiating short pulsed laser. To apply these two different trigger in the single analysis, the programmable digital delay (DG535, Stanford Research, USA) is installed as shown in Fig. 1. The field evaporation is triggered alternately in pulse-by-pulse manner by laser pulse and voltage pulse. The trigger signal is sent to voltage pulser (Hamamatsu Photonics) after the each laser pulse signal to trigger evaporation alternately by laser and voltage at rate of $1000 \mathrm{~Hz}$ for each trigger. "OR" 


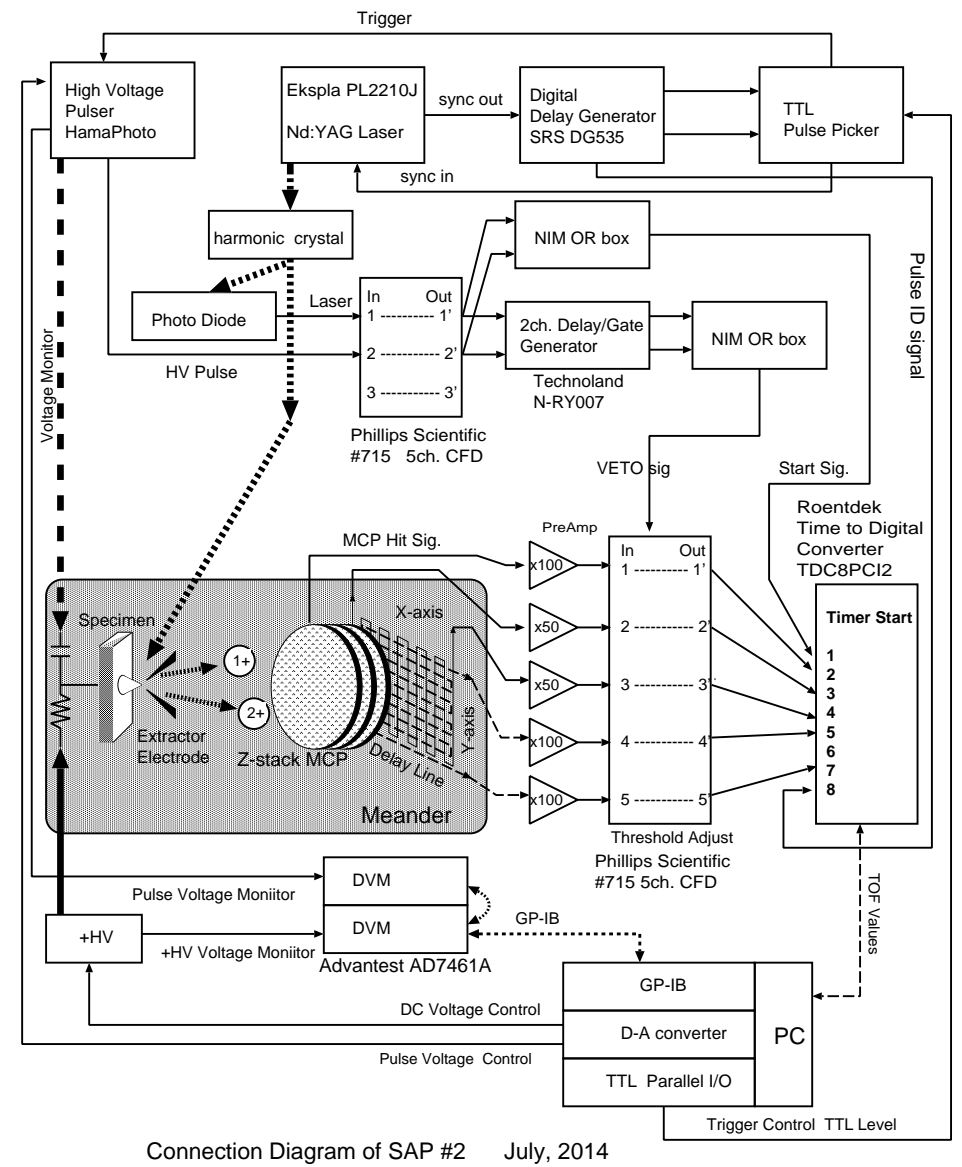

FIG. 1. Schematics of SAP.
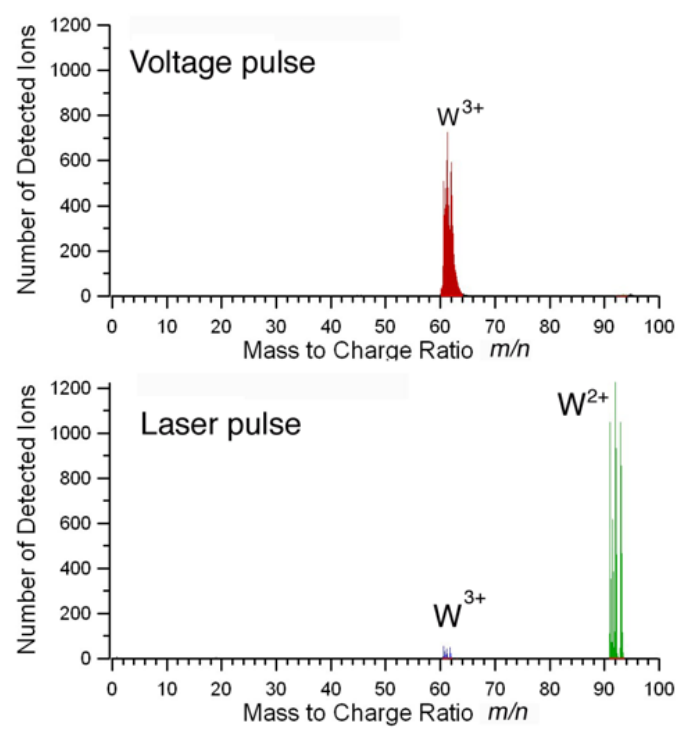

FIG. 2. Histogram of tungsten tip analyzed by dual trigger method. Top: the ions detected by voltage pulse trigger is shown. Bottom: the ions detected by laser pulse trigger is shown.

signal of real trigger timing signal of laser and voltage is generated and sent to digital timer to start the recording sequence. The ID signal (or Flag) of the trigger is also sent to digital timer to discriminate two trigger (laser or voltage). The amplitude of positive DC bias and voltage pulse were automatically controlled by data acquisition system in PC so as keeping the ion detection rate constant typically less than several ions per 100 trigger. The laser pulse amplitude was manually adjusted to keep the same rate as that of voltage pulse.

\section{B. Specimen preparation}

Tungsten wire of $\phi=0.1 \mathrm{~mm}$ was electropolished for bare metallic specimen and also used support of CNT. Single walled nanotube (SWCNT) synthesized by HiPCO process was supplied from Smalley Institute, USA [12]. Small flake of SWCNT was fixed on tungsten tip by silver paste. After the removal of solvent of silver paste in air bath of $400 \mathrm{~K}$ for 5 minutes, SWCNT was soaked in the aqueous solution of L-tryptophan (L-Trp) of $50 \mathrm{mmol} / \mathrm{L}$. The sample dried in the atmosphere at the room temperature was immediately mounted to the UHV chamber for analysis.

\section{RESULTS}

\section{A. Bulk material: electropolished tungsten}

The mass spectra obtained for bare tungsten tip at 200 $\mathrm{K}$ are shown in Fig. 2. The ions detected by voltage pulse and laser pulse are shown in the upper histogram and lower histogram, respectively. The difference of the charge 


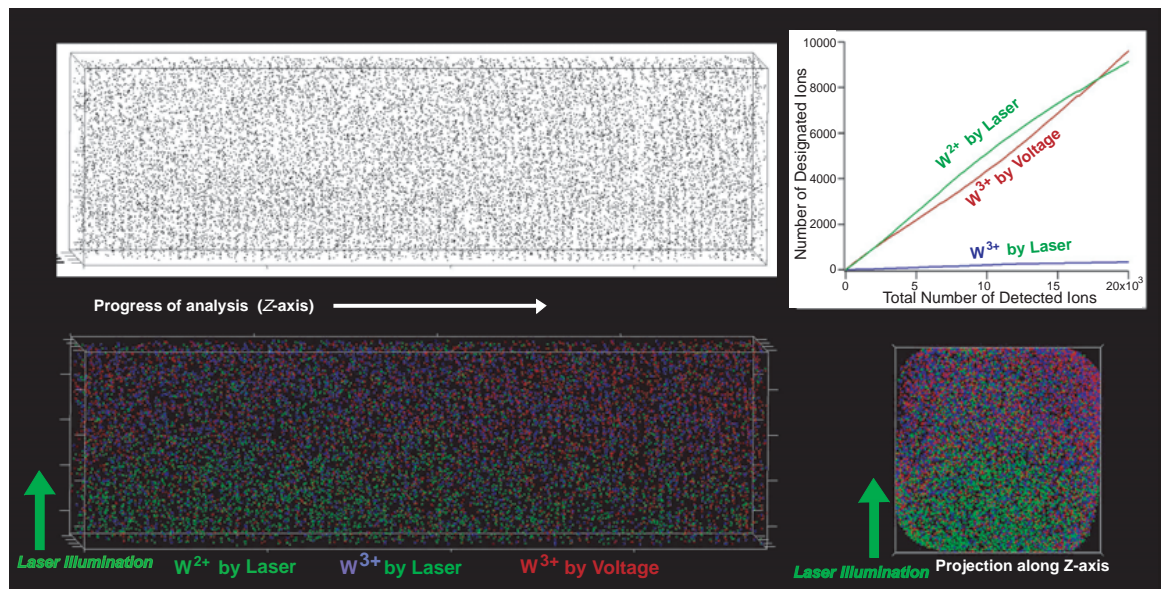

FIG. 3. Analysis of tungsten tip by dual trigger method. Top left: the spatial distribution of ions. The arrow indicates that the evaporation/detection sequence runs from left to right of the figure. The stripes of lattice plane is visible in the vertical direction of the figure. Top right: ladder diagram of the same experiment. The detection rates for voltage and laser trigger are almost balanced during the whole analysis sequence. Bottom left: the spatial distribution of $\mathrm{W}^{2+}$ and $\mathrm{W}^{3+}$ ions along depth axis ( $Z$-axis). Bottom right: the spatial distribution of $\mathrm{W}^{2+}$ and $\mathrm{W}^{3+}$ ions projected along $Z$-axis.

state preference, triply charged for voltage pulsing and doubly charged for laser, can be seen clearly. The ion yield for two triggering method is almost balanced as shown in the ladder diagram of Fig. 3 bottom.

The spatial distribution of ions is plotted in Fig. 3 top. The protocol presented by Bas and Bostel was used to obtain $(x, y, z)$ coordinates for voltage pulse and laser pulse [13]. The trajectory of desorbed ions are determined by the electric field which is generated by the sum of stationary DC bias and the pulse voltage bias "trigger" for voltage pulsing. On the other hand, the electric field is determined only by stationary DC bias for laser pulsing. The $(x, y, z)$ coordinates of the ions are calculated only using DC bias component for both voltage pulsed sequences and laser pulsed sequences and the distribution of both ions are merged in this distribution plot. The pseudo lattice stripes can be seen in the side view of the 3D plot shown in Fig. 3 (top left). Though the density of the ions are spatially homogeneous along depth direction, the voltage triggered ions and the laser triggered ions are segregating in lateral distribution when the plot is projected along depth-direction as sown in Fig. 3 (bottom left and bottom right).

\section{B. Molecular system: amino acid( L-tryptophan) supported on SWCNT}

In this study, L-tryptophan $\mathrm{C}_{11} \mathrm{H}_{12} \mathrm{~N}_{2} \mathrm{O}_{2}$ was chosen as the candidate of molecular system. Figures 4 and 5 show the spectra obtained from the L-tryptophan supported on SWCNT measured at the room temperature. No mass peak above $100 \mathrm{amu}$ which can be assignable to indole ring $\left(\mathrm{C}_{8} \mathrm{H}_{6} \mathrm{~N}-; m / z 116\right)$ is observed here.

When molecules are supported on SWCNT, the substrate $\mathrm{CNT}$ species, $\mathrm{C}^{2+}$ and $\mathrm{C}^{+}$, evaporate prior to the adsorbate fragments $[6,7]$. The components found in Fig. 4 evaporate prior to the components found in Fig. 5 when the analysis is performed using only voltage trigger. The voltage and laser pulse are applied alternately in this measurement, therefore, there seems no sequence between

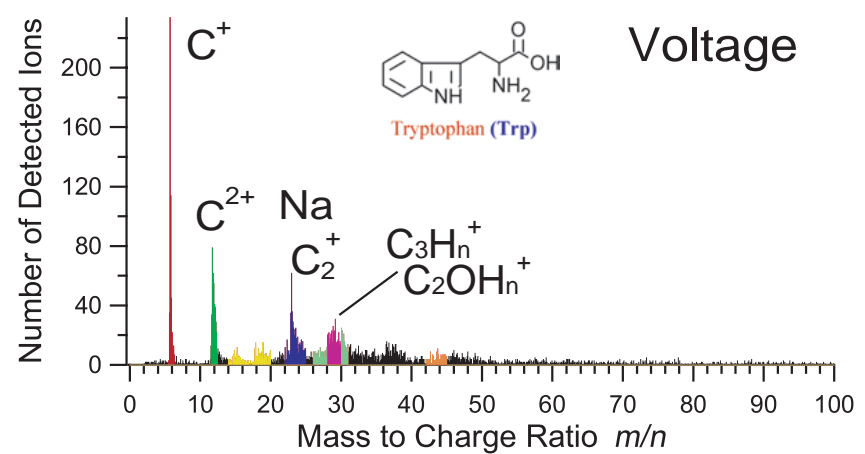

FIG. 4. Mass spectrum of L-Tryptophan/SWCNT. The ions detected by voltage pulse trigger are shown in this spectrum.

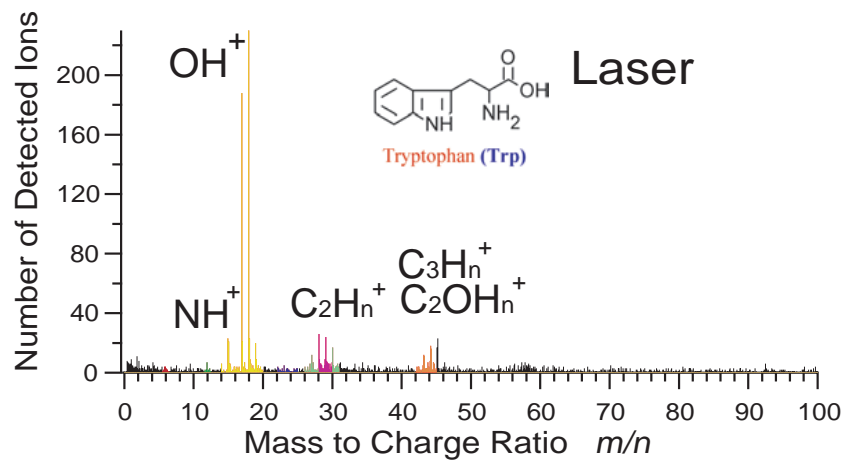

FIG. 5. Mass spectrum of L-Tryptophan/SWCNT. The ions detected by laser pulse trigger are shown in this spectrum.

the components as shown in the ladder diagram of Fig. 6 . They are detected almost simultaneously in the analysis.

\section{DISCUSSION}

The application of voltage pulse and laser pulse alternately (dual trigger) is successful for AP analysis of con- 


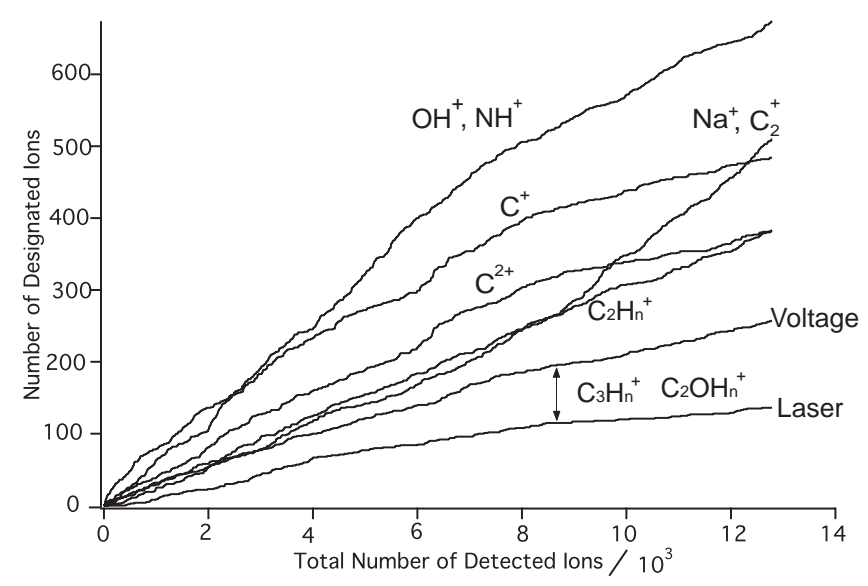

FIG. 6. Ladder diagram of AP analysis of LTryptophan/SWCNT shown in Figure4 and 5.

ventional metallic sample as shown in this study. Though the 3D-information obtained by dual trigger is not equivalent to the orthodox setup using only voltage pulse or only laser pulse because the trajectory of each ion is dynamically changes in voltage triggering (sum of DC bias and voltage pulse) and laser triggering (only DC bias). The results show that the trigger pulses are independent to each other. On the other hand, the angular segregation of the ions by each triggering method shown in Fig. 3 reflects that the shadowing effect of laser irradiation which causes the curvature difference in the irradiated side and the dark side is dynamically compensated by the evaporation from the dark region triggered by voltage pulse
[14].

The mass spectra obtained for L-Tryptophan/SWCNT show that there is preference of evaporation trigger for monoatomic carbon ions from SWCNT (voltage) and for cluster ions of molecular adsorbate L-Tryptophan on SWCNT (laser). It should be noted that the angular distribution of detected ions cannot be directly interpreted as the spatial information of the material in this composite specimen. Though SWCNT can be assume as solid material of nanometer scale, the molecular adsorbate is a mobile species which might be migrate to the vertex of the substrate SWCNT and to be field ionized. The extraction of ion-ion coherence of adsorbate fragment might be an issue of future study.

It is well known that the inhomogeneous or preferential evaporation brings about an artifact in AP analysis. Therefore, the evaporation condition is chosen to keep homogeneous ionization for the optimal spatial information [15]. This applies to solid materials whose spatial information is focused on. But, for the molecular system in which mobile species are supported on solid material, the preferential evaporation might be utilized for the trimming of the support material. The selective evaporation of chosen species might be possible by changing the trigger ratio and sequence between voltage and laser.

\section{ACKNOWLEDGMENTS}

This work was supported by JSPS KAKENHI Grantin-Aid for Scientific Research (C) 24510157.
[1] O. Nishikawa, M. Taniguchi, and M. Ushirozawa, J. Vac. Sci. Technol. B 26, 735 (2008).

[2] O. Nishikawa, M. Taniguchi, and A. Ikai, Appl. Surf. Sci. 256, 1210 (2009).

[3] O. Nishikawa and M. Taniguchi, J. Vac. Sci. Technol. A 27, 1076 (2009).

[4] O. Nishikawa, Y. Ohtani, K. Maeda, M. Watanabe, and K. Tanaka, Mater. Char. 44, 29 (2000).

[5] O. Nishikawa, M. Watanabe, T. Murakami, T. Yagyu, and M. Taniguchi, New Diamond and Frontier Carbon Technol. 13, 257 (2003).

[6] M. Taniguchi, O. Nishikawa, and A. Ikai, Surf. Interface Anal. 44, 721 (2012).

[7] M. Taniguchi and O. Nishikawa, Surf. Interface Anal. 46, 1231 (2014).

[8] E. A. Marquis, M. Bachhav, Y. Chen, Y. Dong, L. M. Gordon, and A. McFarland, Current Opinion in Solid State and Materials Science 17, 217 (2013).

[9] M. K. Miller, et al., Atom Probe Field Ion Microscopy (Oxford Sci. Publications, 1996), p. 86 and p. 258.

[10] N. Mayama, S. Mikami, S. Ito, T. Kaneko, T. Iwata, M. Taniguchi, and M. Owari, e-J. Surf. Sci. Nanotech. 7, 70 (2009).

[11] N. Mayama, T. Terakawa, M. Morita, and M. Owari, e-J. Surf. Sci. Nanotech. 9, 375 (2011).

[12] P. Nikolaev, M. J. Bronikowski, R. K. Bradley, F. Rodmund, D. T. Colbert, K. A. Smith, and R. E. Smalley, Chem. Phys. Lett. 313, 91 (1999).

[13] P. Bas, A. Bostel, B. Deconihout, and D. Blavette, Appl. Surf. Sci. 87/88, 298 (1995).

[14] M. Morita, M. Karasawa, T. Asaka, and M. Owari, J. Surf. Anal. 20, 177 (2014).

[15] D. J. Larson, B. Gault, B. P. Geiser, F. De Geuser, and F. Vurpillot, Current Opinion in Solid State and Materials Science 17, 236 (2013). 